\title{
New and emerging treatments for the prevention of recurrent diverticulitis
}

This article was published in the following Dove Press journal:

Clinical and Experimental Gastroenterology

22 September 201I

Number of times this article has been viewed

\section{Sean T Martin \\ Luca Stocchi \\ Department of Colorectal Surgery, Cleveland Clinic, Cleveland, OH, USA}

Correspondence: Luca Stocchi

Department of Colorectal Surgery,

Digestive Disease Institute, Cleveland

Clinic, 9500 Euclid Avenue, Desk A30,

Cleveland, OH 44195, USA

Tel +I 2164457882

Fax + I 2164458627

Email stocchl@ccf.org

\begin{abstract}
Sigmoid diverticulitis is a common benign condition which carries significant morbidity and socioeconomic burden. This article describes the management of sigmoid diverticulitis with a focus on indications for surgical intervention. The mainstay of management of uncomplicated diverticulitis is broad-spectrum antibiotic therapy. The old surgical dictum that two episodes of sigmoid diverticulitis warranted surgical intervention has been challenged by recently published data. Surgery for diverticulitis thus needs to be tailored to suit individual presentation; patients presenting with recurrent diverticulitis, severe symptoms or debilitating disease impacting patient's quality of life mandate surgical intervention. Complicated diverticular disease typically prompts intervention to resect a diseased, strictured sigmoid colon, fistulizing disease, or a life-threatening colonic perforation. Laterally, minimally invasive surgery has been utilized in the management of this disease and recent data suggests that localized colonic perforation may be managed by laparoscopic peritoneal lavage, without resection. This review focuses discussion on available evidence for contemporary surgical and nonoperative management of diverticulitis.
\end{abstract}

Keywords: sigmoid diverticulitis, colon, laparoscopic peritoneal lavage, surgical intervention

\section{Introduction}

Diverticulitis is a relatively common condition in the developed world which results in a significant number of hospital admissions with subsequent costs to both patients and society at large. The prevalence of the disease increases proportionally with advancing age; however, only a small proportion of patients with diverticulosis will develop symptomatic diverticulitis. It is estimated that $10 \%-25 \%$ of patients with diverticulosis will have an attack of diverticulitis during their lifetime. ${ }^{1}$ It appears that the incidence of the disease is increasing, and patients are tending to present at a younger age. ${ }^{2,3}$ The spectrum of disease ranges from an isolated attack of mild inflammation of sigmoid diverticulae to colonic perforation with fecal peritonitis, a condition with significant morbidity and mortality. This review article will focus on the varied clinical presentation of diverticular disease, the treatment modalities available, the indications for elective and acute surgical intervention, and short and long-term outcomes following surgery.

\section{Risk factors and prevention strategies}

A diet high in intake of fiber, particularly cellulose, is protective against development of diverticular disease as early studies have postulated. ${ }^{4}$ In addition, there is evidence from a large case-control study from the 1980s that the risk of developing 
colonic diverticulosis was increased 1.5 fold in 48,000 male health professionals who consumed red meat. ${ }^{5,6}$ The 20 -year follow-up data from this study (The Health Professionals follow-up study) did not evince dietary intake of nuts, corn, and popcorn in the pathogenesis of diverticulitis, as previously thought. ${ }^{7}$ Obesity has also been implicated in the development of diverticular disease, with relative risk of developing diverticulitis of 1.5-2, depending on whether BMI, waist-to-hip ratio, or waist circumference are considered. ${ }^{8}$ Conversely, physical activity, particularly if vigorous, has been suggested to be protective against developing symptomatic diverticular disease in men and may also protect against bleeding diverticulosis. ${ }^{9,10}$ Some drugs have been suggested as increasing the risk of diverticular disease and its complications; to date only the use of nonsteroidal anti-inflammatory drugs and acetaminophen have been associated with symptoms of severe diverticular disease and its complication of bleeding. ${ }^{11}$

\section{Clinical presentation and investigation of acute diverticulitis}

Patients with acute diverticulitis typically present with acute onset of abdominal pain, localized in the left lower quadrant. Peritoneal irritation may cause localized tenderness with voluntary guarding and rebound found on clinical examination. Occasionally, if a patient has a long, redundant loop of sigmoid colon, the patient may present with right lower quadrant pain as the redundant loop crosses the midline to the right side. Acute diverticulitis should particularly be considered in the differential when an older patient presents with right lower quadrant pain. Classically, patients will have a fever and laboratory studies may show a leukocytosis (predominantly neutrophilia) with elevation of inflammatory markers.

The contemporary mainstay of diagnosis is Computed Tomography (CT) of the abdomen and pelvis; it remains an invaluable adjunct to clinical impression, helps to rule out differential diagnoses, grade the severity of disease, and guide clinical management. We generally recommend against colonoscopy in the acute setting because of the risk of perforating an already inflamed colon with gas insufflation. However, in selected patients in whom the diagnosis is unclear, to rule out pathology such as colon cancer, acute ischemia, or pseudomembranous colitis a gentle flexible sigmoidoscopy may be undertaken.

\section{Evolution in the classification systems used for acute diverticulitis}

There are two main classification modalities for grading the severity of diverticulitis. The more traditional Hinchey Classification grades sigmoid diverticulitis based on findings at surgery ${ }^{12}$ (see Table 1) and was first described in 1978. The major flaw of the Hinchey classification is that it is not applicable to the majority of patients with diverticulitis who can be managed with antibiotics and do not require surgery. Several modifications of the Hinchey grading system have therefore been proposed. Among them, a European consensus conference on diverticular disease has proposed a subclassification of Hinchey grade II diverticulitis, divided into grade IIA, corresponding to a distant abscess amenable to percutaneous drainage and grade IIB, which is a complex abscess with or without an associated fistula. ${ }^{13}$ Another recent advancement in the classification of sigmoid diverticulitis has been introduced by Ambrosetti and colleagues who described the severity of diverticulitis based on CT findings as moderate or severe diverticulitis ${ }^{14}$ (see Table 2). Moderate disease is present when inflammation of the pericolic fat is associated with sigmoid colon wall thickness greater than $5 \mathrm{~mm}$. Severe disease is moderate disease plus at least one of either abscess, free extraluminal gas, or contrast extravasation from the colon. ${ }^{15}$ Chautems et al reported their experience with acute diverticulitis diagnosed by CT in a cohort of 118 patients, followed for a median of 9.5 years. Their data showed that young age $(<50)$ and severe diverticulitis diagnosed on CT scan were independent predictors of poor outcome ( $P=0.007$ and $P=0.003$, respectively). Using this data the authors advocate offering elective surgery to young patients with severe disease on CT scan. ${ }^{16}$

Table I Hinchey classification of acute diverticulitis and its modifications

\begin{tabular}{lll}
\hline & Hinchey classification ${ }^{12}$ & Köhler modification $^{13}$ \\
\hline Stage I & Pericolic abscess confined by the mesocolon & Pericolic abscess \\
Stage II & Pelvic abscess, distant from area of inflammation & $\begin{array}{l}\text { Ila distant abscess amenable to percutaneous drainage } \\
\text { Ilb complex abscess with/without associated fistula }\end{array}$ \\
Stage III & $\begin{array}{l}\text { Generalized peritonitis resulting from pericolic/pelvic abscess rupture } \\
\text { into peritoneal cavity }\end{array}$ & Generalized purulent peritonitis \\
Stage IV & Fecal peritonitis resulting from free perforation of colonic diverticulum & Fecal peritonitis \\
\hline
\end{tabular}


Table 2 Ambrosetti classification ${ }^{15}$

\begin{tabular}{ll}
\hline Moderate diverticulitis & Severe diverticulitis \\
\hline Localized sigmoid colon wall & Moderate diverticulitis plus any of: \\
thickening $(>5 \mathrm{~mm})$ & $\begin{array}{l}\text { Abdominopelvic abscess } \\
\text { Free extraluminal gas } \\
\text { Extraluminal contrast extravasation }\end{array}$ \\
Inflammation localized & \\
to pericolic fat & \\
\hline
\end{tabular}

\section{Management of uncomplicated acute diverticulitis}

In the event of localized sigmoid diverticulitis, with inflammation of the sigmoid colon, the basic tenet of management is administration of broad-spectrum antibiotics. Typically, younger patients who are systemically well with no comorbidity can be managed with oral antibiotics in the outpatient setting. However, older patients, patients with signs of systemic toxicity or significant comorbidity may warrant hospital admission for intravenous antibiotics. However, admission to hospital with acute diverticulitis requiring intravenous antibiotics remains associated with good outcomes. ${ }^{17,18}$ After initiation of intravenous antibiotic therapy one should see an appropriate drop in white blood cell count and temperature. ${ }^{18,19}$ The vast majority of patients with uncomplicated diverticulitis will recover without incident. Current studies are focusing on the identification of possible predictors of patients who are at higher risk of recurrence. Hall et al, in following a cohort of 672 patients after their first attack of uncomplicated diverticulitis for a mean follow-up of 48 months, found an overall recurrence rate of $36 \%$ at 5-year follow-up. Predictors of recurrence included family history, long segment of diseased colon $(>5 \mathrm{~cm})$, and history of retroperitoneal abscess at first presentation. ${ }^{20}$ A small proportion of patients will fail to respond to nonoperative management and experience worsening symptoms. This group of patients may require surgery during the same hospital stay or an expedited elective procedure performed 6 weeks after the acute attack of diverticulitis.

In the majority of patients who recover from an acute attack and are discharged from the hospital, it is critical to exclude other conditions, particularly malignancy, on follow-up. Thus, if the patient has not had a recent colonoscopy it is advisable to schedule one after resolution of the acute attack.

\section{Evolution in the indications for elective surgery}

For many years, the traditional surgical teaching was that an elective sigmoid colectomy should be advised after a second attack of uncomplicated diverticulitis. This principle was based on the assumption that after two episodes of diverticulitis, subsequent disease attacks were inevitable and that surgical resection removed the risk of recurrence presenting acutely with a potentially life-threatening colonic perforation. However, more recent data has challenged the traditional indications for elective resection. Chapman and colleagues reported that patients with more than two prior episodes of diverticulitis were not at increased risk of developing complicated diverticulitis (defined as diverticulitis with perforation, obstruction, abscess, fistula, bleeding, or phlegmon). ${ }^{21}$ Their data demonstrated that morbidity and mortality rates in patients with recurrent diverticulitis were equivalent to that seen in patients who presented with complicated diverticulitis at first presentation. Other studies have confirmed that clinical presentation of free perforation mostly occurs as the first episode of disease and that avoidance of surgery after two episodes is not associated with an increase in emergency surgery and may result in reduced health care costs. ${ }^{22-24}$ The indication for colectomy should therefore not be made based on the potential risk of free perforation. In this respect, the American Society of Colon and Rectal Surgeons (ASCRS) guidelines for elective sigmoid colectomy recommend that "the number of attacks of uncomplicated diverticulitis is not necessarily an overriding factor in defining the appropriateness of surgery". Rather, the decision to operate on uncomplicated disease should be individualized taking into account patient age, medical comorbidity, frequency and severity of attack(s), and persistence of symptoms after resolution of the acute infection. ${ }^{25}$ As a result, the number of patients undergoing elective resection for acute, uncomplicated diverticulitis is falling. Data from the National Inpatient Sample evaluating 685,390 hospital discharges with a diagnosis of acute diverticulitis over a 15 -year time period ending in 2005 indicates that the number of patients with diverticulitis per 1000 hospital discharges increased but the proportion of patients undergoing colectomy for uncomplicated diverticulitis significantly decreased from $17.9 \%$ in 1991 to $13.7 \%$ in 2005. Over the study period the proportion of patients with diverticular abscess increased from $5.9 \%$ to $9.6 \%$ but the percentage of patients with free perforation was unchanged at $1.5 \%$ and the number of perforations/abscesses treated by colectomy declined from $71 \%$ to $55.5 \% .{ }^{3}$ Occasionally patients with recurrent acute diverticulitis may present with symptoms of large bowel obstruction due to stricturing disease, a sequela of recurrent attacks of inflammation that heal by fibrosis. Alternatively, a patient may present with symptoms of fistulizing disease to the bladder, vagina, or 
uterus. Colovesical fistulae can present with pneumaturia. The patient describes passing gas bubbles and fecal sediment per urethra and may give a recent history of recurrent urinary tract infections and dysuria. Many radiologic modalities exist for the diagnostic evaluation of a colovesical fistula; contrast CT of abdomen/pelvis, cystoscopy, contrast enema, and the poppy seed test have all been utilized. Typically, most patients present with classic symptoms of pneumaturia and fecaluria. Melchior and colleagues analyzed their experience with 49 patients diagnosed with colovesical fistula secondary to diverticular disease and found that of these, $71 \%$ presented with pneumaturia and 51\% admitted to fecaluria. Comparing the various diagnostic modalities they found the poppy seed test to have the greatest sensitivity, with $94.6 \%$ (35/37) of patients having a positive test. In contrast, CT picked up $61 \%$, MRI $60 \%$, cystogram $16.7 \%$, contrast enema $35.7 \%$, cystoscopy $10.2 \%$, and colonoscopy $8.5 \%$ of fistulae. ${ }^{26}$ Garcea et al similarly found that most patients presented with symptoms classically attributable to fistulizing disease between the colon and bladder. $90.1 \%$ of their cohort of 90 patients had either pneumaturia or fecaluria. However, they found cystoscopy to be the most sensitive predictor of a fistula (46.2\%) followed by barium enema $(20.1 \%) .{ }^{27}$

Colovaginal fistulae are often encountered against a background of prior hysterectomy where a redundant inflamed sigmoid colon lies on top of the apex of the vagina. On inserting a speculum into the vagina it may be possible to identify a fistulous opening at the apex of the vagina. Colouterine fistulae are rare and usually manifest as crampy lower abdominal pain with purulent vaginal discharge. The sigmoid colon may also fistulize to the small bowel, right or transverse colon, and in a thin person a colocutaneous fistula may ensue.

\section{Elective surgical management of uncomplicated diverticular disease}

The goal of surgery is to remove the diseased sigmoid colon in its entirety, fashioning an anastomosis between the soft, proximal remnant left colon and the upper rectum recognized by the confluence of the teniae. Failure to resect the entire sigmoid colon is associated with a four-fold increased likelihood of developing recurrent diverticulitis. ${ }^{28,29}$ Takedown of the splenic flexure is often required, although not mandatory, to facilitate a tension-free anastomosis. Identification of the ureter is mandatory prior to vessel ligation. Ligation of the inferior mesenteric artery at the origin is not absolutely necessary and might be associated with a period of anorectal malfunction (as long as 6 months) characterized by diarrhea, urgency to defecate, tenesmus, or inability to discriminate between gas and stool. ${ }^{30}$ There is no strong data to support the results of this study as quality of life and functional outcomes after sigmoidectomy are generally excellent. ${ }^{31}$ At times the degree of inflammation in the left lower quadrant may be significant. Perioperative ureteric stenting should be considered in such cases. If an inflammatory phlegmon is encountered it is possible to identify the ureter proximally, often as far cephalad as the renal hilum and follow it distally. This allows safe delineation of the ureter in relation to the inflammatory process, allowing safe mobilization and division of the colon. In such patients, we have a low threshold for a proximal diverting loop ileostomy. Some debate remains regarding the optimal timing of surgery in acute diverticulitis. Some centers advocate operating on the same hospital admission to reduce hospital stay and possible re-admissions with acute diverticulitis in the window period prior to planned surgery. ${ }^{32}$ Natarajan et al, in reviewing their series of laparoscopic sigmoid colectomy for diverticular disease, did not find any association between timing of surgery, complication rate, operative time, recovery period, or conversion to open. ${ }^{33}$ On the other hand, other series have indicated that early surgery was associated with a significant increase in conversion rate $(37.7 \%$ vs $12.9 \%, P<0.001)$ and longer hospitalization (13.5 vs 10.5 days; $P<0.001){ }^{34}$ A prospective German study evaluating the optimal timing of laparoscopic sigmoid resection for uncomplicated and complicated diverticular disease showed that patients having early surgery (4-8 days after initiation of antibiotic therapy, $\mathrm{n}=244$ ) incurred in an increased number of conversions $(9.7 \%$ vs $0.9 \%)$, increased minor morbidity $(25.9 \%$ vs $12.9 \%$ ) and wound infection rates (16.4\% vs $4.6 \%)$ than patients who had delayed surgery. ${ }^{35}$ Based on this data we continue favoring delaying elective surgery by an interval of 4-6 weeks after the latest disease episode.

\section{Evolution in operative approach - from open surgery to minimally invasive techniques}

With the advent of minimally invasive surgery, the laparoscopic and single incision approaches are being increasingly utilized. Many data support that the laparoscopic approach to diverticular disease is associated with accelerated postoperative recovery. A meta-analysis of 19 studies comparing open and laparoscopic sigmoid colectomy has shown equivalence in the incidence of medical complications, rehospitalization or reoperation. In addition, the laparoscopic approach was associated with fewer wound complications $(P<0.05)$, 
blood transfusions $(P<0.01)$ and postoperative ileus rates $(P<0.01) .{ }^{36}$ Furthermore, data from our group has previously indicated that laparoscopic surgery is cost-effective. ${ }^{37}$ A prospective, randomized, single-blinded comparison of open and laparoscopic sigmoid colectomy recently published confirms that the laparoscopic approach is associated with a $30 \%$ reduction in duration of postoperative ileus and hospital stay; the median duration of procedure was 165 minutes in the laparoscopy group and 110 minutes in the open group $(P<0.0001)$. In contrast with this, the median delay between surgery and first bowel movement was 76 hours in the laparoscopy group vs 105 hours in the open group $(P<0.0001)$. The median score for maximal pain assessed by a visual analog scale was 4 in the laparoscopy group vs 5 in the open group $(P=0.05)$. Finally, the median duration of hospital stay was 5 days in the laparoscopy group vs 7 days in the open group $(P<0.0001) .{ }^{38}$ Another prospective randomized controlled trial, the Sigma trial, also showed similar benefits for laparoscopic surgery over open resection. Laparoscopic resection again took longer $(P=0.0001)$ but was associated with decreased intraoperative blood loss. Moreover, there were significantly more major complications in the open sigmoid colectomy group $(9.6 \%$ vs $25.0 \% ; P=0.038)$. Patients undergoing laparoscopic resection also reported less pain, required reduced doses of systemic analgesia, returned home earlier, and experienced a significantly better quality of life. ${ }^{39}$ Very little data exists regarding Hand Assisted Laparoscopic Surgery (HALS), other than a systematic review and metaanalysis of HALS and laparoscopic colorectal surgery in general, which found HALS to be associated with reduction in both operating time and conversion rates, particularly pertaining to diverticulitis. ${ }^{40}$ HALS might be particularly suitable for extensively inflamed, adherent, or fistulizing sigmoid colon disease. More recently, several short series on single incision laparoscopic surgery (SILS) sigmoid colectomy for diverticular disease have been published; ${ }^{41,42}$ however, these series are small and the procedure is still in its embryonic stages, thus we cannot comment on its utility in diverticular disease other than to say that it has promise.

\section{Outcomes following surgical resection of sigmoid diverticular disease}

Outcomes following laparoscopic or open sigmoid colectomy for diverticular disease are generally considered to be excellent. However, the actual data on quality of life following sigmoid colectomy for diverticular disease remain limited. The Cleveland Clinic Florida analyzed 57 patients who had a sigmoid colectomy for diverticular disease (23 laparoscopic, 34 open). The SF-36 Health Survey, which measures eight different health-quality domains, was generally excellent with no difference observed in the laparoscopic group when compared to patients having open surgery. Only small bowel obstruction and incisional herniae were associated with a lower SF-36 score. ${ }^{43}$ Forgione et al described outcomes in a cohort of 46 patients who had sigmoid colectomy performed for diverticular disease, 45 of whom had laparoscopic procedure. All patients were assessed preoperatively and at 3,6 , and 12 months postoperatively regarding quality of life and bowel symptomatology (gastrointestinal quality of life index [GIQLI]). The questionnaire, developed by Eypasch, ${ }^{44}$ contains up to 36 items, scored on a five-point Likert scale (range $0-144$, higher score $=$ better QOL), in which additional modules, specified by the particular chronic gastrointestinal disease, supplement a set of core questions. Quality of life significantly improved in the group, with 36 patients having an increase in their GIQLI greater than 10 points. These improvements were evident at 3 months and maintained at 1 year follow-up. The improvement was due to increments in gastrointestinal symptoms and was most significant in those with lowest preoperative scoring. However, five of 46 patients had a deterioration in GIQLI without a precipitating event. There was no impairment in urinary and sexual function postoperatively. ${ }^{28}$ Recurrence rates after elective resection of sigmoid diverticular disease should be less than 5\% after appropriate resection. ${ }^{45,46}$ However, some patients may return with recurrent symptoms, with no objective clinical, radiologic, or endoscopic evidence of either recurrent diverticulitis or anastomotic stricture. Egger et al found in their cohort of 162 patients who had a sigmoid colectomy for diverticular disease that $25 \%$ of patients evaluated at follow-up had recurrent symptoms. However, none of these patients had recurrent diverticulitis and recurrent symptoms were independent of indication for surgery (complicated vs uncomplicated diverticulitis) or operative approach (open vs laparoscopic). ${ }^{46}$ It is possible that at least in some cases the symptoms are attributable to Irritable Bowel Syndrome but not all cases of recurrent symptoms following surgery for sigmoid diverticulitis are easily explainable. These occurrences underscore the importance of CT scan to accurately establish the diagnosis of sigmoid diverticulitis.

\section{Complicated diverticular disease}

Complications of diverticular disease include stricture, fistula, bleeding, perforation, and abscess. There is some data to suggest that patients in lower socioeconomic groups are more 
likely to present emergently with complications of disease; similarly, they are less likely to have surgery. ${ }^{47,48}$ Elective surgery is indicated for complicated diverticular disease in a good-risk patient. On the other hand, when medical comorbid conditions make surgery hazardous, a nonoperative approach may be adopted. The operative approach to complicated sigmoid diverticulitis depends on the disease presentation in each individual patient. Emergency surgery is required to manage perforated diverticular disease with generalized peritonitis; fistulizing and stricturing disease typically can instead be dealt with in the elective setting. Diverticular abscesses may require initial percutaneous drainage (Hinchey Grade I, II; Ambrosetti severe diverticulitis ), depending on the size of the abscess. There is no uniform acceptance of which abscesses require drainage based on size alone; however, the available data would suggest that abscesses less than $3 \mathrm{~cm}$ can be initially approached with antibiotics alone and even abscesses of 3-4 cm in maximal diameter are likely to respond to antibiotic therapy alone. On the other hand, abscesses greater than $4 \mathrm{~cm}$ are likely to require percutaneous drainage ${ }^{49}$ and when the diameter exceeds $6.5 \mathrm{~cm}$ and is associated with a temperature greater than $101^{\circ} \mathrm{F}$, failure of antibiotic therapy can definitely be anticipated. ${ }^{50}$ An alternative view has been proposed questioning the validity of radiologic drainage of diverticular abscess. A case-control study from Switzerland suggests that percutaneous drainage does not derive any benefit over antibiotic therapy alone. A total of 34 patients with a diverticular abscess (mean diameter $6 \mathrm{~cm}$, range 3-18 cm) had percutaneous CT-guided drainage and were compared with 32 patients with an abscess (mean diameter $4 \mathrm{~cm}$, range $3-10 \mathrm{~cm}$ ) who were treated with antibiotics as radiologic drainage was not technically feasible. Only 6 of 32 patients failed the antibiotic regimen (failure being defined as needing an emergency operation, worsening sepsis, or recurrence of abscess within 4 weeks of therapy) as compared to 11 of 34 patients in the radiologic intervention group (33\% vs $19 \%$, NS). ${ }^{51}$ These data question the need for radiologic intervention and warrant further investigation. It is our practice to initially treat diverticular abscesses with radiologic drainage when technically feasible, particularly for abscesses larger than $5 \mathrm{~cm}$ and pelvic abscesses.

\section{Surgery for complicated diverticular disease}

Elective surgery for complicated disease is typically for a stricture, fistula, or phlegmon. A stricture of the sigmoid colon secondary to chronic diverticulitis may be impossible to differentiate from sigmoid carcinoma and can present acutely as large bowel obstruction. In this setting, surgery is necessary. The operative approach of choice is patient dependent. If the patient is young, and can tolerate a big operation, sigmoid colectomy with primary anastomosis is advised. Inclusion of a proximal diverting ileostomy is at the surgeon's discretion. In performing a primary anastomosis in an acutely obstructed bowel fecal loading is often encountered proximal to the stricture and on-table colonic lavage via an appendicostomy or enterotomy in the terminal ileum may be necessary. Occasionally, a grossly dilated left colon may be deemed unsuitable for anastomosis and a Hartmann's procedure is preferable. Similarly, in an older, infirm patient it may be advisable to perform a proximal diversion to relieve the obstruction to shorten anesthetic time and potential complications. It may be possible to do a staged resection at a later date when the patients' medical condition has been optimized. In those patients considered poor surgical candidates, at high mortality risk, some authors would advocate use of endoscopically placed metallic stents to relieve symptoms. However, reobstruction and perforation are major and not infrequent complications of endoscopic stent placement for sigmoid strictures. ${ }^{52}$

Fistulizing disease from the sigmoid colon represents a strong indication for operative intervention. Fistulizing disease is most commonly seen between the sigmoid colon and bladder. ${ }^{26,53}$ Colectomy for colovesical fistulae can be challenging. Typically, the sigmoid colon is densely adherent to the dome of the bladder and the pelvic sidewall. In these circumstances one must remain cognizant that the left ureter may be pulled medially into the inflammatory phlegmon. Many data support laparoscopy as the approach of choice to fistulizing disease, recognizing that the conversion rate may be higher and operating time longer, in this subset of patients. ${ }^{51-57}$ Caution needs to be exercised in female patients with fistulae to the genital tract as there is a significant risk of ureteric injury, up to $5 \%$ in published series. ${ }^{58}$ HALS may be a procedure which minimizes intraoperative complications as the use of a hand in the peritoneal cavity can facilitate finger fracture of a diseased, inflamed colon from the bladder and retroperitoneum, obviating the need to use electrocautery devices in these areas, where injudicious use can cause injury to adjacent structures.

\section{Surgery for generalized peritonitis secondary to diverticular perforation}

Perforation of a sigmoid colon diverticulum with generalized peritonitis is a surgical emergency associated with significant mortality. Traditionally, the operation of choice for these 
patients was a Hartmann's procedure whose contemporary modification formally consists of sigmoid colectomy with end descending colostomy and oversewing of the rectal stump. This operation has also been performed laparoscopically. ${ }^{59,60}$ In practice, patients are often significantly compromised during surgery and an expedited resection may benefit a septic, hemodynamically compromised patient. This involves simply resecting the perforated segment of the sigmoid colon, (a "perforectomy"), exteriorizing the proximal end as a colostomy, and oversewing the distal segment. The remainder of the sigmoid colon is excised at the time of colostomy reversal. The morbidity and mortality for a Hartmann's procedure remains high which likely reflects the nature of the underlying pathology and patient condition rather than the procedure. ${ }^{60,61}$ Despite a large body of literature to support primary anastomosis, with or without proximal diversion, ${ }^{62-65}$ the Hartmann's procedure is not yet obsolete and remains an important part of the surgeon's armamentarium. It may be possible, in select circumstances, to resect the perforated, diseased segment of colon and restore intestinal continuity with a primary colorectal anastomosis. This procedure may require on-table colonic lavage via an appendicostomy, which prolongs the operation and involves liberation of the splenic and occasionally, the hepatic flexure. A proximal ileostomy may be utilized, based on surgeon preference. ${ }^{63-65}$ A large Dutch prospective trial, the LADIES trial, ${ }^{66}$ has started to recruit patients to either a Hartmann's procedure or a primary anastomosis for management of Hinchey III/IV diverticulitis and we eagerly await their findings. A major consideration when performing a Hartmann's procedure is that a significant proportion of patients will not have the colostomy reversed for a variety of reasons. ${ }^{67-69}$ In addition, colostomy reversal is associated with an appreciable morbidity. ${ }^{70,71}$ It is possible that the reversal operation can be performed using a minimally invasive approach, with slight decrease in postoperative morbidity. $^{72}$

\section{Management of sigmoid diverticulitis in the immunocompromised patient}

Patients who are immunocompromised or immunosuppressed after transplant surgery are particularly susceptible to developing diverticulitis. Consistent data suggest that the attacks of diverticulitis tend to be more virulent, more likely to be associated with perforation ${ }^{73}$ and have a higher morbidity and mortality than in the general population. ${ }^{7-76}$ It is practice at our institution to advise early surgical intervention in these patients, either soon after resolution of the first attack or during the same hospital stay. Many other centers practice these recommendations, although no good data exists to support this aggressive surgical approach to a very high-risk group of patients. ${ }^{77,78}$ We routinely fashion a defunctioning loop ileostomy after elective sigmoid resection in these patients. ${ }^{78}$ Occasionally, patients present with uncomplicated diverticulitis while awaiting transplant. It is our policy to adopt an expectant approach to these patients because of their poor state of health and consider sigmoidectomy in case of recurrent diverticulitis only after transplant. With respect to HIV-positive patients there is data suggesting that lower CD4+ counts are associated with poorer outcomes and increased morbidity rates after surgery. ${ }^{79}$

\section{The evolution of alternative surgical management for Hinchey III diverticulitis: laparoscopic lavage}

Laparoscopic peritoneal lavage was first described by O'Sullivan and colleagues in eight patients with purulent generalized peritonitis, none of whom required further surgical intervention during 48 months of follow-up. This original report from 1996 prompted a prospective, multi-institutional Irish study of 100 patients with purulent peritonitis following diverticular perforation. Patients had laparoscopic evaluation of the peritoneal cavity, lavage with $4 \mathrm{~L}$ of warm saline solution and no resection. The selective use of drains was left to the individual surgeon's discretion with feculent peritonitis or a visible opening in the sigmoid as contraindications to this approach. Morbidity and mortality rates were $4 \%$ and $3 \%$, respectively. Only two patients $(2.2 \%)$ required subsequent percutaneous drainage of a pelvic abscess. At median follow-up of 36 months, only two patients $(2.2 \%)$ presented with recurrent diverticulitis ${ }^{80}$ This study prompted several other groups to assess the feasibility of laparoscopic lavage for Hinchey III diverticulitis. An Australian group has shown similarly encouraging data, with eight out of 27 patients $(29.6 \%)$ treated with laparoscopic lavage developing recurrent complicated diverticulitis requiring elective surgical resection. A further $29.6 \%$ (8/27) had an uncomplicated postoperative course with early, planned resection. The remaining patients $(11 / 27,40.8 \%)$ had no further sequelae at a mean follow-up of 20 months. ${ }^{81}$ A retrospective, comparative French study evaluated the outcomes of patients with Hinchey III diverticulitis treated by laparoscopic lavage $(n=24)$ compared with those in whom resection and anastomosis $(\mathrm{n}=35)$ was undertaken. The results showed that the median hospital stay was lower in patients treated by laparoscopic peritoneal lavage ( 8 vs 17 days, $P<0.0001$ ). Twenty-five patients in the laparoscopic peritoneal lavage group subsequently underwent 
Table 3 Suggested treatment algorithm for acute diverticulitis

\begin{tabular}{|c|c|c|c|}
\hline \multicolumn{2}{|c|}{ Acute uncomplicated diverticulitis } & \multicolumn{2}{|c|}{ Acute complicated diverticulitis } \\
\hline Presentation & Management & Presentation & Management \\
\hline \multirow[t]{3}{*}{ LLQ pain } & $\begin{array}{l}\text { Oral antibiotics } \\
\text { Admission for iv antibiotics in older } \\
\text { patients with comorbidity/signs of sepsis }\end{array}$ & $\begin{array}{l}\text { Diverticulitis with associated } \\
\text { abscess }\end{array}$ & $\begin{array}{l}\text { Admission, iv antibiotics if abscess }<5 \mathrm{~cm} \\
\text { If no response, or abscess }>5 \mathrm{~cm}, \\
\text { percutaneous abscess drainage }\end{array}$ \\
\hline & & Purulent peritonitis & $\begin{array}{l}\text { Laparoscopic lavage or } \\
\text { laparotomy, sigmoid colectomy with/ } \\
\text { without proximal diversion }\end{array}$ \\
\hline & & Fecal peritonitis & Hartmann's procedure \\
\hline
\end{tabular}

Abbreviations: LLQ, left lower quadrant; iv, intravenous.

elective laparoscopic resection, with only one case converted to laparotomy. Cumulative surgical morbidity ( $16 \%$ vs $37.5 \%$, $P=0.0507)$ and hospital stay (14 vs 23 days, $P<0.0001$ ) was less in the laparoscopic peritoneal lavage group. ${ }^{82}$ A noteworthy, potential advantage of laparoscopic lavage is that it may reduce the number of patients requiring an ostomy by converting an emergency resection with proximal stoma/colostomy to an elective laparoscopic sigmoid colectomy with primary anastomosis. In addition, a significant proportion of patients having successful laparoscopic lavage may not require definitive surgery at all. The true value of this new technique will become evident once the results of a randomized controlled trial, the Lapland Study (http://clinicaltrials.gov/ct2/show/ study/NCT01019239?term=lapland\&rank=1), an Irish-based multi-institutional prospective RCT, become known.

A suggested paradigm for management of Sigmoid Diverticulitis is shown in Table 3.

\section{Alternative approach in the medical management of diverticulitis: anti-inflammatory agents}

Several authors have suggested that sigmoid diverticulitis is a form of inflammatory bowel disease which could benefit from treatment with anti-inflammatory agents. For example, a combination of rifamixin and mesalazine for 10 days to induce remission in symptomatic uncomplicated diverticular disease followed by mesalazine alone for 8 weeks was tested in a study cohort of 90 patients. ${ }^{83}$ This regimen was associated with a statistically significant improvement in disease symptoms with $78 \%$ of patients being completely asymptomatic after 8 weeks of therapy. It has been suggested based on the results of a series of 268 patients that higher doses of mesalazine (800 mg BID) are associated with a statistically significant improvement in symptoms when compared to lower doses of mesalazine (400 mg BID) and rifamixin. Probiotics combined with mesalazine have also emerged as an alternative potential therapeutic strategy in preventing recurrent attacks of diverticulitis. One series reported that treatment with mesalazine and/or lactobacillus casei induced remission in $88 \%$ of their patients at a median follow-up of 2 years. However, cessation of the treatment was associated with relapse of symptoms in all cases. ${ }^{84}$ These studies are limited by small numbers and stronger evidence is needed to support routine use of mesalazine or probiotics in clinical practice.

\section{Conclusion}

Sigmoid diverticulitis is a condition with diverse clinical consequences, ranging from mild inflammation to lifethreatening perforation. The majority of cases resolve with broad-spectrum antibiotic therapy. In regards to immunocompromised patients with diverticulitis we advocate early surgical intervention after the first episode. The indications for elective surgery for uncomplicated diverticulitis should otherwise be tailored for each individual patient. In contrast, most clinicians agree that sigmoidectomy is warranted for complicated diverticular disease. Laparoscopic sigmoidectomy is now accepted as an advantageous alternative to open surgery in select patients. Laparoscopic lavage may be an option for generalized, non-feculent peritonitis.

\section{Disclosure}

The authors declare no conflicts of interest in this work.

\section{References}

1. Boles RS Jr, Jordan SM. The clinical significance of diverticulosis. Gastroenterology. 1958;35(6):579-582.

2. Etzioni DA, Mack TM, Beart RW Jr, Kaiser AM. Diverticulitis in the United States: 1998-2005: changing patterns of disease and treatment. Ann Surg. 2009;249(2):210-217.

3. Ricciardi R, Baxter NN, Read TE, Marcello PW, Hall J, Roberts PL. Is the decline in the surgical treatment for diverticulitis associated with an increase in complicated diverticulitis? Dis Colon Rectum. 2009;52(9):1558-1563.

4. Painter NS, Burkitt DP. Diverticular disease of the colon: a deficiency disease of Western civilization. Br Med J. 1971;2(5759):450-454.

5. Aldoori WH, Giovannucci EL, Rimm EB, Wing AL, Trichopoulos DV, Willett WC. A prospective study of diet and the risk of symptomatic diverticular disease in men. Am J Clin Nutr. 1994;60(5):757-764. 
6. Manousos O, Day NE, Tzonou A, et al. Diet and other factors in the aetiology of diverticulosis: an epidemiological study in Greece. Gut. 1985;26(6):544-549.

7. Strate LL, Liu YL, Syngal S, Aldoori WH, Giovannucci EL. Nut, corn, and popcorn consumption and the incidence of diverticular disease. JAMA. 2008;300(8):907-914.

8. Strate LL, Liu YL, Aldoori WH, Syngal S, Giovannucci EL. Obesity increases the risks of diverticulitis and diverticular bleeding. Gastroenterology. 2009;136(1):115-122.

9. Aldoori WH, Giovannucci EL, Rimm EB, et al. Prospective study of physical activity and the risk of symptomatic diverticular disease in men. Gut. 1995;36(2):276-282.

10. Strate LL, Liu YL, Aldoori WH, Giovannucci EL. Physical activity decreases diverticular complications. Am J Gastroenterol. 2009;104(5): 1221-1230.

11. Aldoori W, Ryan-Harshman M. Preventing diverticular disease. Review of recent evidence on high-fibre diets. Can Fam Physician. 2002;48:1632-1637.

12. Hinchey EJ, Schaal PG, Richards GK. Treatment of perforated diverticular disease of the colon. Adv Surg. 1978;12:85-109.

13. Köhler L, Sauerland S, Neugebauer E. Diagnosis and treatment of diverticular disease: results of a consensus development conference. The Scientific Committee of the European Association for Endoscopic Surgery. Surg Endosc. 1999;13(4):430-436.

14. Ambrosetti P, Jenny A, Becker C, Terrier TF, Morel P. Acute left colonic diverticulitis - compared performance of computed tomography and water-soluble contrast enema: prospective evaluation of 420 patients. Dis Colon Rectum. 2000;43(10):1363-1367.

15. Ambrosetti P, Grossholz M, Becker C, Terrier F, Morel P. Computed tomography in acute left colonic diverticulitis. Br J Surg. 1997; 84(4):532-534.

16. Chautems RC, Ambrosetti P, Ludwig A, Mermillod B, Morel P, Soravia C. Long-term follow-up after first acute episode of sigmoid diverticulitis: is surgery mandatory?: a prospective study of 118 patients. Dis Colon Rectum. 2002;45(7):962-966.

17. Alvarez JA, Baldonedo RF, Bear IG, et al. Presentation, management and outcome of acute sigmoid diverticulitis requiring hospitalization. Dig Surg. 2007;24(6):471-476.

18. Evans J, Kozol R, Frederick W, et al. Does a 48-hour rule predict outcomes in patients with acute sigmoid diverticulitis? J Gastrointest Surg. 2008;12(3):577-582. Epub January 3, 2008.

19. Sra HK, Shipman K, Virk HS. Does a 48-hour rule predict outcomes in patients with acute sigmoid diverticulitis? J Gastrointest Surg 2009;13(10):1892.

20. Hall JF, Roberts PL, Ricciardi R, et al. Long-term follow-up after an initial episode of diverticulitis: what are the predictors of recurrence? Dis Colon Rectum. 2011;54(3):283-288.

21. Chapman JR, Dozois EJ, Wolff BG, Gullerud RE, Larson DR. Diverticulitis: a progressive disease? Do multiple recurrences predict less favorable outcomes? Ann Surg. 2006;243(6):876-880. Discussion 880-883.

22. Ritz JP, Lehmann KS, Frericks B, Stroux A, Buhr HJ, Holmer C. Outcome of patients with acute sigmoid diverticulitis: Multivariate analysis of risk factors for free perforation. Surgery. 2011;149(5):606-613.

23. Anaya DA, Flum DR. Risk of emergency colectomy and colostomy in patients with diverticular disease. Arch Surg. 2005;140(7):681-685.

24. Salem L, Veenstra DL, Sullivan SD, Flum DR. The timing of elective colectomy in diverticulitis: a decision analysis. J Am Coll Surg. 2004;199(6):904-912.

25. Rafferty J, Shellito P, Hyman NH, Buie WD. Standards Committee of American Society of Colon and Rectal Surgeons. Practice parameters for sigmoid diverticulitis. Dis Colon Rectum. 2006;49(7):939-944.

26. Melchior S, Cudovic D, Jones J, Thomas C, Gillitzer R, Thüroff J. Diagnosis and surgical management of colovesical fistulas due to sigmoid diverticulitis. J Urol. 2009;182(3):978-982.

27. Garcea G, Majid I, Sutton CD, Pattenden CJ, Thomas WM. Diagnosis and management of colovesical fistulae; six-year experience of 90 consecutive cases. Colorectal Dis. 2006;8(4):347-352.
28. Benn PL, Wolff BG, Ilstrup DM. Level of anastomosis and recurrent colonic diverticulitis. Am J Surg. 1986;151(2):269-271.

29. Thaler K, Baig MK, Berho M, et al. Determinants of recurrence after sigmoid resection for uncomplicated diverticulitis. Dis Colon Rectum. 2003;46(3):385-388.

30. Sarli L, Pavlidis C, Cinieri FG, et al. Prospective comparison of laparoscopic left hemicolectomy for colon cancer with laparoscopic left hemicolectomy for benign colorectal disease. World J Surg. 2006; 30(3):446-452.

31. Forgione A, Leroy J, Cahill RA, et al. Prospective evaluation of functional outcome after laparoscopic sigmoid colectomy. Ann Surg. 2009;249(2):218-224

32. Wasvary H, Turfah F, Kadro O, Beauregard W. Same hospitalization resection for acute diverticulitis. Am Surg. 1999;65(7):632-635.

33. Natarajan S, Ewings EL, Vega RJ. Laparoscopic sigmoid colectomy after acute diverticulitis: when to operate? Surgery. 2004;136(4):725-730.

34. Zingg U, Pasternak I, Guertler L, Dietrich M, Wohlwend KA, Metzger U. Early versus delayed elective laparoscopic-assisted colectomy in sigmoid diverticulitis: timing of surgery in relation to the acute attack. Dis Colon Rectum. 2007;50(11):1911-1917.

35. Ritz JP, Reissfelder C, Holmer C, Buhr HJ. Results of sigma resection in acute complicated diverticulitis: method and time of surgical intervention. Chirurg. 2008;79(8):753-758.

36. Siddiqui MR, Sajid MS, Qureshi S, Cheek E, Baig MK. Elective laparoscopic sigmoid resection for diverticular disease has fewer complications than conventional surgery: a meta-analysis. Am J Surg. 2010;200(1):144-161.

37. Senagore AJ, Duepree HJ, Delaney CP, Dissanaike S, Brady KM, Fazio VW. Cost structure of laparoscopic and open sigmoid colectomy for diverticular disease: similarities and differences. Dis Colon Rectum. 2002;45(4):485-490.

38. Gervaz P, Inan I, Perneger T, Schiffer E, Morel P. A prospective, randomized, single-blind comparison of laparoscopic versus open sigmoid colectomy for diverticulitis. Ann Surg. 2010;252(1):3-8.

39. Klarenbeek BR, Veenhof AA, Bergamaschi R, et al. Laparoscopic sigmoid resection for diverticulitis decreases major morbidity rates: a randomized control trial: short-term results of the Sigma Trial. Ann Surg. 2009;249(1):39-44.

40. Aalbers AG, Biere SS, van Berge Henegouwen MI, Bemelman WA. Handassisted or laparoscopic-assisted approach in colorectal surgery: a systematic review and meta-analysis. Surg Endosc. 2008;22(8):1769-1780.

41. Champagne BJ, Lee EC, Leblanc F, Stein SL, Delaney CP. Singleincision vs straight laparoscopic segmental colectomy: a case-controlled study. Dis Colon Rectum. 2011;54(2):183-186.

42. Vestweber B, Alfes A, Paul C, Haaf F, Vestweber KH. Single-incision laparoscopic surgery: a promising approach to sigmoidectomy for diverticular disease. Surg Endosc. 2010;24(12):3225-3228.

43. Thaler K, Dinnewitzer A, Mascha E, et al. Long-term outcome and health-related quality of life after laparoscopic and open colectomy for benign disease. Surg Endosc. 2003;17(9):1404-1408.

44. Eypasch E, Williams JI, Wood-Dauphinee S, et al. Gastrointestinal Quality of Life Index: development, validation and application of a new instrument. Br J Surg. 1995;82(2):216-222.

45. Bergamaschi R, Arnaud JP. Anastomosis level and specimen length in surgery for uncomplicated diverticulitis of the sigmoid. Surg Endosc. 1998;12(9):1149-1151.

46. Egger B, Peter MK, Candinas D. Persistent symptoms after elective sigmoid resection for diverticulitis. Dis Colon Rectum. 2008;51(7):1044-1048.

47. Yoo PS, Garg R, Salamone LF, Floch MH, Rosenthal R, Longo WE. Medical comorbidities predict the need for colectomy for complicated and recurrent diverticulitis. Am J Surg. 2008;196(5):710-714.

48. Csikesz NG, Singla A, Simons JP, Tseng JF, Shah SA. The impact of socioeconomic status on presentation and treatment of diverticular disease. J Gastrointest Surg. 2009;13(11):1993-2001.

49. Siewert B, Tye G, Kruskal J, et al. Impact of CT-guided drainage in the treatment of diverticular abscesses: size matters. Am J Roentgenol. 2006;186(3):680-686. 
50. Kumar RR, Kim JT, Haukoos JS, et al. Factors affecting the successful management of intra-abdominal abscesses with antibiotics and the need for percutaneous drainage. Dis Colon Rectum. 2006;49(2):183-189.

51. Brandt D, Gervaz P, Durmishi Y, Platon A, Morel P, Poletti PA. Percutaneous CT scan-guided drainage versus antibiotherapy alone for Hinchey II diverticulitis: a case-control study. Dis Colon Rectum. 2006;49(10):1533-1538.

52. Small AJ, Young-Fadok TM, Baron TH. Expandable metal stent placement for benign colorectal obstruction: outcomes for 23 cases. Surg Endosc. 2008;22(2):454-462.

53. Altman D, Forsgren C, Hjern F, Lundholm C, Cnattingius S, Johansson AL. Influence of hysterectomy on fistula formation in women with diverticulitis. Br J Surg. 2010;97(2):251-257.

54. Engledow AH, Pakzad F, Ward NJ, Arulampalam T, Motson RW. Laparoscopic resection of diverticular fistulae: a 10-year experience. Colorectal Dis. 2007;9(7):632-634.

55. Lee SW, Yoo J, Dujovny N, Sonoda T, Milsom JW. Laparoscopic versus hand-assisted laparoscopic sigmoidectomy for diverticulitis. Dis Colon Rectum. 2006 Apr;49(4):464-469.

56. Bartus CM, Lipof T, Sarwar CM, et al. Colovesical fistula: not a contraindication to elective laparoscopic colectomy. Dis Colon Rectum. 2005;48(2):233-236.

57. Pokala N, Delaney CP, Brady KM, Senagore AJ. Elective laparoscopic surgery for benign internal enteric fistulas: a review of 43 cases. Surg Endosc. 2005;19(2):222-225.

58. Hjern F, Goldberg SM, Johansson C, Parker SC, Mellgren A. Management of diverticular fistulae to the female genital tract. Colorectal Dis. 2007;9(5):438-442.

59. Chouillard E, Maggiori L, Ata T, et al. Laparoscopic two-stage left colonic resection for patients with peritonitis caused by acute diverticulitis. Dis Colon Rectum. 2007;50(8):1157-1163.

60. Issa N, Dreznik Z, Dueck DS, et al. Emergency surgery for complicated acute diverticulitis. Colorectal Dis. 2009;11(2):198-202.

61. Vermeulen J, Akkersdijk GP, Gosselink MP, et al. Outcome after emergency surgery for acute perforated diverticulitis in 200 cases. Dig Surg. 2007;24(5):361-366.

62. Constantinides VA, Heriot A, Remzi F, et al. Operative strategies for diverticular peritonitis: a decision analysis between primary resection and anastomosis versus Hartmann's procedures. Ann Surg. 2007; 245(1):94-103.

63. Trenti L, Biondo S, Golda T, et al. Generalized peritonitis due to perforated diverticulitis: Hartmann's procedure or primary anastomosis? Int J Colorectal Dis. 2011;26(3):377-384.

64. Zingg U, Pasternak I, Dietrich M, Seifert B, Oertli D, Metzger U. Primary anastomosis vs Hartmann's procedure in patients undergoing emergency left colectomy for perforated diverticulitis. Colorectal Dis. 2010;12(1):54-60.

65. Salem L, Flum DR. Primary anastomosis or Hartmann's procedure for patients with diverticular peritonitis? A systematic review. Dis Colon Rectum. 2004;47(11):1953-1964.

66. Swank HA, Vermeulen J, Lange JF, et al. Dutch Diverticular Disease (3D) Collaborative Study Group. The ladies trial: laparoscopic peritoneal lavage or resection for purulent peritonitis and Hartmann's procedure or resection with primary anastomosis for purulent or faecal peritonitis in perforated diverticulitis (NTR2037). BMC Surg. 2010;10:29.
67. van de Wall BJ, Draaisma WA, Schouten ES, Broeders IA, Consten EC. Conventional and laparoscopic reversal of the Hartmann procedure: a review of literature. J Gastrointest Surg. 2010;14(4):743-752.

68. Oomen JL, Cuesta MA, Engel AF. Reversal of Hartmann's procedure after surgery for complications of diverticular disease of the sigmoid colon is safe and possible in most patients. Dig Surg. 2005;22(6):419-425.

69. Keck JO, Collopy BT, Ryan PJ, Fink R, Mackay JR, Woods RJ. Reversal of Hartmann's procedure: effect of timing and technique on ease and safety. Dis Colon Rectum. 1994;37(3):243-248.

70. Leong QM, Koh DC, Ho CK. Emergency Hartmann's procedure: morbidity, mortality and reversal rates among Asians. Tech Coloproctol. 2008;12(1):21-25.

71. Berry AR, Turner WH, Mortensen NJ, Kettlewell MG. Emergency surgery for complicated diverticular disease. A five-year experience. Dis Colon Rectum. 1989;32(10):849-854.

72. Siddiqui MR, Sajid MS, Baig MK. Open vs laparoscopic approach for reversal of Hartmann's procedure: a systematic review. Colorectal Dis. 2010;12(8):733-741.

73. Chapman J, Davies M, Wolff B, et al. Complicated diverticulitis: is it time to rethink the rules? Ann Surg. 2005;242(4):576-581. Discussion 581-583.

74. Leonardi MJ, Jamil KG, Hiscox B, Ross D, Hiatt JR. Abdominal surgery after lung transplantation. Am Surg. 2010;76(10):1130-1134.

75. Hwang SS, Cannom RR, Abbas MA, Etzioni D. Diverticulitis in transplant patients and patients on chronic corticosteroid therapy: a systematic review. Dis Colon Rectum. 2010;53(12):1699-1707.

76. Goldberg HJ, Hertz MI, Ricciardi R, Madoff RD, Baxter NN, Bullard KM. Colon and rectal complications after heart and lung transplantation. JAm Coll Surg. 2006;202(1):55-61.

77. Hesterberg R, Müller F, Schmidt WU, Möslein G, Lammers B. Sigmoid diverticulitis in immunosuppressive drug therapy. Chirurg. 1994;65(10):873-876. German.

78. Aydin HN, Remzi FH. Diverticulitis: when and how to operate? Dig Liver Dis. 2004;36(7):435-445.

79. Albaran RG, Webber J, Steffes CP. CD4 cell counts as a prognostic factor of major abdominal surgery in patients infected with the human immunodeficiency virus. Arch Surg. 1998;133(6):626-631.

80. Myers E, Hurley M, O’Sullivan GC, Kavanagh D, Wilson I, Winter DC. Laparoscopic peritoneal lavage for generalized peritonitis due to perforated diverticulitis. Br J Surg. 2008;95(1):97-101.

81. White SI, Frenkiel B, Martin PJ. A ten-year audit of perforated sigmoid diverticulitis: highlighting the outcomes of laparoscopic lavage. Dis Colon Rectum. 2010;53(11):1537-1541.

82. Karoui M, Champault A, Pautrat K, Valleur P, Cherqui D, Champault G. Laparoscopic peritoneal lavage or primary anastomosis with defunctioning stoma for Hinchey 3 complicated diverticulitis: results of a comparative study. Dis Colon Rectum. 2009;52(4):609-615.

83. Tursi A, Brandimarte G, Giorgetti GM, Elisei W. Mesalazine and/or Lactobacillus casei in maintaining long-term remission of symptomatic uncomplicated diverticular disease of the colon. Hepatogastroenterology. 2008;55(84):916-920.

84. Comparato G, Fanigliulo L, Cavallaro LG, et al. Prevention of complications and symptomatic recurrences in diverticular disease with mesalazine: a 12-month follow-up. Dig Dis Sci. 2007;52(11):2934-2941.
Clinical and Experimental Gastroenterology

\section{Publish your work in this journal}

Clinical and Experimental Gastroenterology is an international, peerreviewed, open access journal, publishing all aspects of gastroenterology in the clinic and laboratory, including: Pathology, pathophysiology of gastrointestinal disease; Investigation and treatment of gastointestinal disease; Pharmacology of drugs used in the alimentary tract;

\section{Dovepress}

Immunology/genetics/genomics related to gastrointestinal disease. This journal is indexed on CAS. The manuscript management system is completely online and includes a very quick and fair peer-review system. Visit http://www.dovepress.com/testimonials.php to read real quotes from published authors. 